\title{
KdV cnoidal waves in a traffic flow model with periodic boundaries
}

\section{Article}

\section{Accepted Version}

Creative Commons: Attribution-Noncommercial-No Derivative Works 4.0

Hattam, L. L. (2017) KdV cnoidal waves in a traffic flow model with periodic boundaries. Physica D: Nonlinear Phenomena, 348. pp. 44-53. ISSN 0167-2789 doi:

https://doi.org/10.1016/j.physd.2017.02.010 Available at https://centaur.reading.ac.uk/69302/

It is advisable to refer to the publisher's version if you intend to cite from the work. See Guidance on citing.

To link to this article DOI: http://dx.doi.org/10.1016/j.physd.2017.02.010

Publisher: Elsevier

All outputs in CentAUR are protected by Intellectual Property Rights law, including copyright law. Copyright and IPR is retained by the creators or other copyright holders. Terms and conditions for use of this material are defined in the End User Agreement.

\section{www.reading.ac.uk/centaur}

\section{CentAUR}

Central Archive at the University of Reading

Reading's research outputs online 


\title{
$\mathrm{KdV}$ cnoidal waves in a traffic flow model with periodic boundaries
}

\author{
Laura Hattam *
}

\begin{abstract}
An optimal-velocity (OV) model describes car motion on a single lane road. In particular, near to the boundary signifying the onset of traffic jams, this model reduces to a perturbed Korteweg-de Vries (KdV) equation using asymptotic analysis. Previously, the KdV soliton solution has then been found and compared to numerical results (see Muramatsu and Nagatani (1999)). Here, we instead apply modulation theory to this perturbed KdV equation to obtain at leading order, the modulated cnoidal wave solution. At the next order, the Whitham equations are derived, which have been modified due to the equation perturbation terms. Next, from this modulation system, a family of spatially periodic cnoidal waves are identified that characterise vehicle headway distance. Then, for this set of solutions, we establish the relationship between the wave speed, the modulation term and the driver sensitivity. This analysis is confirmed with comparisons to numerical solutions of the OV model. As well, the long-time behaviour of these solutions is investigated.
\end{abstract}

\section{Introduction}

The study of traffic flow has uncovered some interesting phenomena such as the propagation of nonlinear density waves representing congestion. To determine traffic behaviour, a variety of modelling techniques are used, which include the application of car following, cellular automation, gas kinetic and hydro dynamical models. Refer to Nagatani (2002) for a discussion of the different methods.

Here, we concentrate on a car following model that governs vehicle motion on a single lane road with periodic boundaries. The OV model proposed by Newell (1961) is applied, which is

$$
\frac{d x_{j}(t+\tau)}{d t}=V\left(x_{j+1}(t)-x_{j}(t)\right),
$$

where $x_{j}(t)$ is the position of car $j$ at time $t, \tau$ is the delay time of the driver, $V$ is the optimal velocity and $j=0,1,2, \ldots, N$ for $N$ cars on the road.

*l.hattam@reading.ac.uk, Department of Mathematics and Statistics, University of Reading, UK 
We will examine (1) when it reduces to a perturbed $\mathrm{KdV}$ equation. This occurs only within a certain stability zone, which is referred to as 'metastable'. It is well-known that the unperturbed $\mathrm{KdV}$ equation has the travelling wave solution, the cnoidal wave of modulus $m$, where $m \in(0,1)$ (see Korteweg and de Vries (1895)). If $m \rightarrow 1$, this becomes the soliton solution.

Muramatsu and Nagatani (1999) explored this traffic flow problem with open boundaries. They derived a perturbed KdV equation from an OV model and then obtained the KdV soliton solution. This result was compared to numerical simulations with good agreement. As well, these solutions were shown to disappear after some time. Zhu and Dai (2008) instead applied periodic boundary conditions to study the metastable dynamics. Numerically they found large amplitude waves with narrow peaks of both upward and downward form. Nonlinear theory was next used to find the perturbed $\mathrm{KdV}$ equation and the soliton solution was highlighted to explain the numerical waves exhibited. Note however that this solution does not satisfy periodic boundaries and therefore, further analysis is needed.

Additionally, Yu et al. (2010) and Zhou et al. (2014) performed numerical examinations of traffic OV models. This revealed steady travelling waves in the metastable zone that are similar in form to the KdV cnoidal wave of modulus $m$. The modulation term appears to be constant here since the wave amplitude, mean height and period remain fixed over the domain. Therefore, it is possible that other KdV solutions do occur in the OV model besides the soliton. Hence, further asymptotic work is required to establish the connection between the numerically observed travelling density waves and the nonlinear analysis. To achieve this, we will apply modulation theory to the perturbed KdV equation so that the entire family of possible solutions to the reduced traffic model are obtained.

Whitham (1974) developed modulation theory for the KdV equation, which was a multi-scale method that gave modulated wavetrain solutions. A system of first order partial differential equations describing the modulations was also found, now known as the 'Whitham Equations'. Gurevich and Pitaevskii (1987) extended the modulation theory to include Burgers damping, where the Whitham equations with additional terms to account for friction were formed. Myint and Grimshaw (1995) instead incorporated an arbitrary damping term, and then considered three different types of damping to analyse the subsequent wavetrain solutions. Later, Kamchatnov (2004) presented a generalised technique to obtain modified Whitham equations for the perturbed KdV equation. These previous studies will be used here. Then, by assuming steady travelling waves exist, we will demonstrate how a perturbed Whitham system can be reduced to a single differential equation for the modulation term.

This paper concentrates on the identification of steady solutions of the modulation equations, in particular, when the modulation term is fixed since these appear numerically. More specifically, in Section 2, we outline the traffic OV model and the asymptotic analysis used to then obtain a 
perturbed KdV equation. Next, in Section 3, modulation theory is applied to this equation and as a result, the modified Whitham equations are derived. Then, steady solutions of these equations are pursued in Section 4. This analysis is next related to the traffic problem in Section 5. Lastly, in Section 6, numerical simulations of the OV model are compared to the asymptotic solutions. As well, the long-time dynamics are examined.

\section{Traffic Flow Model}

We outline the transformation of (1) into a perturbed KdV equation within the metastable zone. Firstly, a Taylor series expansion of (1) gives

$$
\frac{d^{2} \Delta x_{j}}{d t^{2}}=\hat{a}\left(V\left(\Delta x_{j+1}(t)\right)-V\left(\Delta x_{j}(t)\right)-\frac{d \Delta x_{j}}{d t}\right)
$$

where $\hat{a}=1 / \tau$ is the driver's sensitivity and $\Delta x_{j}=x_{j+1}-x_{j}$ is the vehicle headway. Bando et al. (1995) proposed this model, as well as the optimal velocity function,

$$
V\left(\Delta x_{j}(t)\right)=\frac{v_{\max }}{2}\left(\tanh \left(\Delta x_{j}-h_{c}\right)+\tanh \left(h_{c}\right)\right),
$$

where $h_{c}$ is the safety distance and $v_{\max }$ is the maximal velocity. We choose $v_{\max }=2$ and $h_{c}=4$ for convenience.

Ge et al. (2005) discussed three OV models that describe car motion. Here, their Model B is applied, which uses (2) and (3), and has the linear stability criteria

$$
\tau \leq \tau_{s}=\frac{1}{2 V^{\prime}(h)}
$$

If this condition is satisfied, then the steady solution $\Delta x_{j}(t)=h$ is stable, where $h$ is the uniform headway. Additionally, the curve given by $\tau=\tau_{s}$ is labelled the 'neutral stability line' as it represents the boundary between no traffic jams and jams.

Next, Ge et al. (2005) detailed the application of an asymptotic method to reduce (2) to a perturbed KdV equation, which used the change of variables

$$
x=-\epsilon \sqrt{\frac{6}{V^{\prime}(h)}}\left(j+V^{\prime}(h) t\right), \quad \bar{t}=\epsilon^{3} \sqrt{\frac{6}{V^{\prime}(h)}} t, \quad \epsilon^{2}=1-\left(\tau / \tau_{s}\right), \quad 0<\epsilon \ll 1,
$$

and let

$$
\Delta x_{j}(t)=h+\frac{\epsilon^{2}}{V^{\prime \prime}(h)} u
$$

Consequently, (2) became

$$
u_{\bar{t}}+\nu u u_{x}+\lambda u_{x x x}+\epsilon \bar{V}(u)=0,
$$


where

$$
\bar{V}(u)=\mu u_{x x}+\gamma u_{x x x x}+\eta\left(u^{2}\right)_{x x},
$$

and

$$
\lambda=1, \quad \nu=1, \quad \mu=-\sqrt{\frac{3 V^{\prime}(h)}{2}}, \quad \gamma=\frac{3}{2} \sqrt{\frac{3}{2 V^{\prime}(h)}}, \quad \eta=\frac{1}{2} \sqrt{\frac{3}{2 V^{\prime}(h)}} .
$$

This is a perturbed $\mathrm{KdV}$ equation as $\epsilon$ is small. We have introduced here the parameters $\lambda, \nu, \mu, \gamma, \eta$ so that the perturbation analysis in Sections 3 and 4 is generalised. Then, this is related to our traffic problem in Sections 5 and 6.

Since $0<\epsilon \ll 1, \tau$ is chosen such that the solutions to (7) are positioned very close to the neutral stability line, however, they will still satisfy (4). Therefore, as $t$ becomes very large, the headway will tend to steady state $h$. This region is classified as metastable. Soliton density waves within this zone have been shown numerically to propagate for long times and eventually disappear (refer to Muramatsu and Nagatani (1999)). We however will demonstrate that a large set of long-time persisting cnoidal wave solutions exist. Here, it is only periodic boundaries that are considered, therefore spatially periodic solutions are sought. It should be noted that reference throughout to the spatial domain corresponds to $j \in[0, N]$. So, to implement periodic boundary conditions, we ensure

$$
\Delta x_{0}(t)=\Delta x_{N}(t),\left.\quad \frac{\partial \Delta x_{j}(t)}{\partial j}\right|_{j=0}=\left.\frac{\partial \Delta x_{j}(t)}{\partial j}\right|_{j=N}, \quad t \geq 0
$$

\section{The Modulation Equations}

Previous studies, such as Myint and Grimshaw (1995) and Kamchatnov (2004), have derived modulation theory for the perturbed KdV equation. From these workings, we know that if

$$
u(x, \bar{t})=u_{0}(\theta, X, T)+O(\epsilon)
$$

and

$$
\theta=\frac{1}{\epsilon} \Theta(X, T), \quad X=\epsilon x, \quad T=\epsilon \bar{t}, \quad \omega=-\Theta_{T}, \quad k=\Theta_{X}, \quad c=\omega / k,
$$

where $c$ is the wave speed, then

$$
u_{0}=a b+d+a \operatorname{cn}^{2}\left(\beta\left(\theta-\theta_{0}\right) ; m\right),
$$


where

$$
\begin{aligned}
& a=\frac{12 \lambda}{\nu}(m k \beta)^{2}, \\
& d=\frac{c}{\nu}-\frac{a}{3 m^{2}}\left(2-m^{2}-3 \frac{E(m)}{K(m)}\right), \\
& b=\frac{1-m^{2}}{m^{2}}-\frac{E(m)}{m^{2} K(m)}, \\
& \beta=K(m) / P .
\end{aligned}
$$

This is the cnoidal wave solution with period $2 P$ in $\theta$, where $P$ is a fixed constant. The parameters $m, a, b, d, k, \theta_{0}, \beta$ are slowly varying, dependent on the slow variables $X$ and $T$. The function cn is the Jacobi elliptic function and $K(m), E(m)$ are the elliptic integrals of the first and second kind respectively. Additionally, this solution satisfies

$$
\frac{\lambda k^{2}}{\nu} u_{0, \theta}^{2}=2 \hat{D}+2 \hat{C} u_{0}+U u_{0}^{2}-\frac{1}{3} u_{0}^{3}
$$

where $\hat{C}$ and $\hat{D}$ are integration constants and $U=c / \nu$. These constants can be expressed in terms of the cnoidal wave parameters,

$$
\begin{aligned}
& \hat{C}=-\frac{1}{3}(a b+d)^{3}+\frac{1}{2} U(a b+d)^{2}-\frac{a^{2}}{6 m^{2}}\left(1-m^{2}\right)(a b+d), \\
& \hat{D}=\frac{1}{2}(a b+d)^{2}-U(a b+d)+\frac{a^{2}}{6 m^{2}}\left(1-m^{2}\right) .
\end{aligned}
$$

The Whitham modulation equations for the perturbed KdV equation are also determined using previous findings (see Myint and Grimshaw (1995) and Kamchatnov (2004)). This is a third order system written in terms of the Riemann invariants $r_{1}, r_{2}, r_{3}$, which describe the slow variation of $(12)$,

$$
\frac{\partial r_{i}}{\partial T}+Q_{i} \frac{\partial r_{i}}{\partial X}=M_{i}
$$

where

$$
Q_{i}=\nu U+\frac{\nu}{6} \frac{k}{\partial_{r_{i}} k}, \quad i=1,2,3, \quad U=\frac{1}{6}\left(r_{1}+r_{2}+r_{3}\right), \quad m^{2}=\frac{r_{2}-r_{1}}{r_{3}-r_{1}},
$$


and

$$
\begin{aligned}
M_{1}=- & \frac{k}{2 P\left(\partial_{r_{1}} k\right)\left(r_{3}-r_{1}\right)\left(r_{2}-r_{1}\right)} \times \\
& \left(\int_{-P}^{P} u_{0} \bar{V}\left(u_{0}\right) d \theta-\frac{1}{2}\left(r_{2}+r_{3}-r_{1}\right) \int_{-P}^{P} \bar{V}\left(u_{0}\right) d \theta\right), \\
M_{2}=- & \frac{k}{2 P\left(\partial_{r_{2}} k\right)\left(r_{3}-r_{2}\right)\left(r_{1}-r_{2}\right)} \times \\
& \left(\int_{-P}^{P} u_{0} \bar{V}\left(u_{0}\right) d \theta-\frac{1}{2}\left(r_{1}+r_{3}-r_{2}\right) \int_{-P}^{P} \bar{V}\left(u_{0}\right) d \theta\right), \\
M_{3}=- & \frac{k}{2 P\left(\partial_{r_{3}} k\right)\left(r_{1}-r_{3}\right)\left(r_{2}-r_{3}\right)} \times \\
& \left(\int_{-P}^{P} u_{0} \bar{V}\left(u_{0}\right) d \theta-\frac{1}{2}\left(r_{1}+r_{2}-r_{3}\right) \int_{-P}^{P} \bar{V}\left(u_{0}\right) d \theta\right) .
\end{aligned}
$$

Now, we apply this analysis to the perturbed $\mathrm{KdV}$ equation (7), where $\bar{V}$ is defined by (8). So, using (14),

$$
\begin{aligned}
\frac{1}{2 P} \int_{-P}^{P} u_{0} \bar{V}\left(u_{0}\right) d \theta= & \frac{1}{2 P} \int_{-P}^{P} u_{0}\left(\mu k^{2} u_{0, \theta \theta}+\gamma k^{4} u_{0, \theta \theta \theta \theta}+\eta k^{2}\left(u_{0}^{2}\right)_{\theta \theta}\right) d \theta \\
= & -\mu k^{2} \frac{1}{2 P} \int_{-P}^{P} u_{0, \theta}^{2} d \theta+\gamma k^{4} \frac{1}{2 P} \int_{-P}^{P} u_{0} u_{0, \theta \theta \theta \theta} d \theta \\
& +\eta k^{2} \frac{1}{2 P} \int_{-P}^{P} u_{0}\left(-\frac{2 \lambda k^{2}}{\nu} u_{0, \theta \theta \theta \theta}+2 U u_{0, \theta \theta}\right) d \theta \\
= & -\left(\mu k^{2}+2 U \eta k^{2}\right) \frac{1}{2 P} \int_{-P}^{P} u_{0, \theta}^{2} d \theta+\left(\gamma k^{4}-\frac{2 \lambda \eta}{\nu} k^{4}\right) \frac{1}{2 P} \int_{-P}^{P} u_{0, \theta \theta}^{2} d \theta .
\end{aligned}
$$

Next, omitting the details, it can be shown that by manipulating (14),

$$
\begin{aligned}
\frac{1}{2 P} \frac{\lambda k^{2}}{\nu} \int_{-P}^{P} u_{0, \theta}^{2} d \theta & =\frac{2}{5}(3 \hat{D}+2 \hat{C} d+U(\hat{C}+U d)), \\
\frac{1}{2 P} \frac{\lambda^{2} k^{4}}{\nu^{2}} \int_{-P}^{P} u_{0, \theta \theta}^{2} & =\frac{1}{7}\left(6 \hat{D} d+8 \hat{C}^{2}+U\left(-6 \hat{D}+6 \hat{C} d+2 U \hat{C}+2 U^{2} d\right)\right) .
\end{aligned}
$$

As well, since $u_{0}$ and its derivatives with respect to $\theta$ are periodic over $2 P$, then

$$
\int_{-P}^{P} \bar{V}\left(u_{0}\right) d \theta=\int_{-P}^{P}\left(\mu k^{2} u_{0, \theta \theta}+\gamma k^{4} u_{0, \theta \theta \theta \theta}+\eta k^{2}\left(u_{0}^{2}\right)_{\theta \theta}\right) d \theta=0 .
$$

As a result,

$$
M_{i}=-\frac{k \int_{-P}^{P} u_{0} \bar{V}\left(u_{0}\right) d \theta}{2 P \partial_{r_{i}} k \prod_{i \neq j}\left(r_{i}-r_{j}\right)},
$$


where

$$
\begin{aligned}
\frac{1}{2 P} \int_{-P}^{P} u_{0} \bar{V}\left(u_{0}\right) d \theta= & -\left(\mu k^{2}+2 U \eta k^{2}\right) \frac{2 \nu}{5 \lambda k^{2}}(3 \hat{D}+2 \hat{C} d+U(\hat{C}+U d)) \\
& +\left(\gamma k^{4}-\frac{2 \lambda \eta}{\nu} k^{4}\right) \frac{\nu^{2}}{7 \lambda^{2} k^{4}}\left(6 \hat{D} d+8 \hat{C}^{2}+U\left(-6 \hat{D}+6 \hat{C} d+2 U \hat{C}+2 U^{2} d\right)\right) .
\end{aligned}
$$

Thus, the Whitham system for the traffic flow problem outlined in Section 2 has been identified.

\section{Steady Solutions}

The differential equations have been derived that govern the modulation of our leading order cnoidal wave solution (12). We now seek steady solutions to the system (16)-(17) by setting the wave speed, $c$, to a constant. Here, the workings of El et al. (2005) are followed, where steady solutions were found to the fourth order Whitham system for the Kaup-Boussinesq-Burgers equation. This method is now adapted to analyse our third order system.

Initially, the equation perturbation terms, $\bar{V}$, are undefined so that the analysis is generalised. Consequently, a technique is detailed to reduce any perturbed third order modulation system to a single differential equation for the modulation term (assuming $\int_{-P}^{P} \bar{V}\left(u_{0}\right) d \theta=0$ ).

Now, if $c$ is some constant,

$$
\frac{\partial}{\partial T}=-\omega \frac{d}{d \Theta}, \quad \frac{\partial}{\partial X}=k \frac{d}{d \Theta} .
$$

and

$$
\frac{\partial r_{i}}{\partial T}+Q_{i} \frac{\partial r_{i}}{\partial X}=\left(-k \nu U+k \nu U+k \tilde{Q}_{i}\right) \frac{d r_{i}}{d \Theta}=M_{i},
$$

where

$$
\tilde{Q}_{i}=Q_{i}-\nu U=\frac{\nu}{6} \frac{k}{\partial_{r_{i}} k}, \quad M_{i}=-\frac{k \tilde{M}}{2 \partial_{r_{i}} k \prod_{i \neq j}\left(r_{i}-r_{j}\right)}, \quad \tilde{M}=2\left(\frac{1}{2 P} \int_{-P}^{P} u_{0} \bar{V}\left(u_{0}\right) d \theta\right) .
$$

Therefore,

$$
\frac{d r_{i}}{d \Theta}=\frac{M_{i}}{k \tilde{Q}_{i}}=\frac{\hat{M}}{\Pi_{i \neq j}\left(r_{i}-r_{j}\right)}, \quad \hat{M}=-\frac{3}{k \nu} \tilde{M}
$$

Now let

$$
P(r)=\Pi_{i=1}^{3}\left(r-r_{i}\right)=r^{3}-s_{1} r^{2}+s_{2} r-s_{3},
$$

where

$$
s_{1}=r_{1}+r_{2}+r_{3}, \quad s_{2}=r_{1} r_{2}+r_{1} r_{3}+r_{2} r_{3}, \quad s_{3}=r_{1} r_{2} r_{3} .
$$

Then,

$$
\frac{d s_{1}}{d \Theta}=\frac{d}{d \Theta}\left(r_{1}+r_{2}+r_{3}\right)=\left(\sum_{i=1}^{3} \frac{1}{\Pi_{i \neq j}\left(r_{i}-r_{j}\right)}\right) \hat{M}=0
$$


As well,

$$
\frac{d s_{2}}{d \Theta}=\frac{d}{d \Theta}\left(r_{1} r_{2}+r_{1} r_{3}+r_{2} r_{3}\right)=\left(\sum_{i=1}^{3} \frac{\sum_{j}^{\prime} r_{j}}{\Pi_{i \neq j}\left(r_{i}-r_{j}\right)}\right) \hat{M}=0 .
$$

Hence, $s_{1}$ and $s_{2}$ are any real constants. Next,

$$
\frac{d s_{3}}{d \Theta}=\frac{d}{d \Theta}\left(r_{1} r_{2} r_{3}\right)=s_{3}\left(\sum_{i=1}^{3} \frac{1}{r_{i}} \cdot \frac{1}{\Pi_{i \neq j}\left(r_{i}-r_{j}\right)}\right) \hat{M}=s_{3} \cdot \frac{1}{s_{3}} \cdot \hat{M}=\hat{M} .
$$

Note that the three identities

$$
\sum_{i=1}^{n} \frac{1}{\Pi_{i \neq j}\left(r_{i}-r_{j}\right)}=0, \quad \sum_{i=1}^{n} \frac{\sum_{j}^{\prime} r_{j}}{\Pi_{i \neq j}\left(r_{i}-r_{j}\right)}=0, \quad \sum_{i=1}^{n} \frac{1}{r_{i}} \cdot \frac{1}{\Pi_{i \neq j}\left(r_{i}-r_{j}\right)}=\frac{(-1)^{n-1}}{\Pi_{i}^{n} r_{i}}
$$

were used to determine $s_{i, \Theta}$, which are from El et al. (2005).

Now, we know that

$$
\begin{aligned}
\frac{\lambda k^{2}}{\nu} u_{0, \theta}^{2} & =-\frac{1}{3}\left(u_{0}-\frac{1}{2}\left(r_{1}+r_{2}-r_{3}\right)\right)\left(u_{0}-\frac{1}{2}\left(r_{1}+r_{3}-r_{2}\right)\right)\left(u_{0}-\frac{1}{2}\left(r_{2}+r_{3}-r_{1}\right)\right), \\
& =2 \hat{D}+2 \hat{C} u_{0}+U u_{0}^{2}-\frac{1}{3} u_{0}^{3} .
\end{aligned}
$$

This becomes, if $u_{0}=-\tilde{u}_{0}+s_{1} / 2=-\tilde{u}_{0}+\left(r_{1}+r_{2}+r_{3}\right) / 2$ and using $(20)$,

$$
\begin{aligned}
\frac{\lambda k^{2}}{\nu} \tilde{u}_{0, \theta}^{2} & =\frac{1}{3}\left(\tilde{u}_{0}-r_{3}\right)\left(\tilde{u}_{0}-r_{2}\right)\left(\tilde{u}_{0}-r_{1}\right) \\
& =\frac{1}{3} P\left(\tilde{u}_{0}\right)=\frac{1}{3}\left(\tilde{u}_{0}^{3}-s_{1} \tilde{u}_{0}^{2}+s_{2} \tilde{u}_{0}-s_{3}\right), \\
& =2 \hat{D}+2 \hat{C}\left(-\tilde{u}_{0}+s_{1} / 2\right)+U\left(-\tilde{u}_{0}+s_{1} / 2\right)^{2}-\frac{1}{3}\left(-\tilde{u}_{0}+s_{1} / 2\right)^{3} .
\end{aligned}
$$

Equating like terms of (23), we find

$$
U=\frac{s_{1}}{6}, \quad \hat{C}=-\frac{s_{2}}{6}+\frac{s_{1}^{2}}{24}, \quad \hat{D}=-\frac{s_{3}}{6}+\frac{s_{1}}{48}\left(4 s_{2}-s_{1}^{2}\right) .
$$

As an aside, the integration constant, $\hat{C}$, can be written as a function of $a$ and $m$ only using (13b) and (13c). After some detail, (15a) takes the form

$$
\hat{C}=\frac{a^{2}}{18 m^{4}}\left(m^{4}-m^{2}+1\right)-\frac{s_{1}^{2}}{72} .
$$

Therefore, from (24),

$$
-\frac{s_{2}}{6}+\frac{s_{1}^{2}}{24}=\frac{a^{2}}{18 m^{4}}\left(m^{4}-m^{2}+1\right)-\frac{s_{1}^{2}}{72} .
$$

Rearranging this,

$$
a(m)=\kappa^{1 / 2} \sqrt{\frac{18 m^{4}}{m^{4}-m^{2}+1}},
$$


where

$$
\kappa=\frac{s_{1}^{2}}{18}-\frac{s_{2}}{6}>0,
$$

which is some constant since $s_{1}, s_{2}$ are constants. From (25), (13b) takes the form

$$
d(m)=\frac{s_{1}}{6}-\frac{\sqrt{\kappa}}{3} \sqrt{\frac{18}{m^{4}-m^{2}+1}}\left(2-m^{2}-3 \frac{E(m)}{K(m)}\right) .
$$

Next, from (13c), (25) and (27), we can express $\hat{D}$ as a function of $m$ only. With some simplification, (15b) can be written

$$
\hat{D}=\frac{\sqrt{2}}{3}\left(\frac{\kappa}{m^{4}-m^{2}+1}\right)^{3 / 2}\left(-2 m^{6}+3 m^{4}+3 m^{2}-2\right)+\frac{s_{1} s_{2}}{36}-\frac{11 s_{1}^{3}}{1296} .
$$

Combining this with our second definition for $\hat{D}$, see $(24)$,

$$
-\frac{s_{3}}{6}+\frac{s_{1}}{48}\left(4 s_{2}-s_{1}^{2}\right)=\frac{\sqrt{2}}{3}\left(\frac{\kappa}{m^{4}-m^{2}+1}\right)^{3 / 2}\left(-2 m^{6}+3 m^{4}+3 m^{2}-2\right)+\frac{s_{1} s_{2}}{36}-\frac{11 s_{1}^{3}}{1296} .
$$

This gives us an expression for $s_{3}$ in terms of $m$ only,

$$
s_{3}(m)=-\frac{1}{27}\left(\frac{s_{1}^{2}-3 s_{2}}{m^{4}-m^{2}+1}\right)^{3 / 2}\left(-2 m^{6}+3 m^{4}+3 m^{2}-2\right)+\frac{s_{1} s_{2}}{3}-\frac{2 s_{1}^{3}}{27},
$$

and then,

$$
\frac{d s_{3}}{d m}=\frac{\left(s_{1}^{2}-3 s_{2}\right)^{3 / 2} m^{3}\left(m^{2}-1\right)}{\left(m^{4}-m^{2}+1\right)^{5 / 2}} .
$$

Thus, from (22),

$$
\frac{d m}{d \Theta}=-\frac{3}{k \nu}\left(\frac{d s_{3}}{d m}\right)^{-1} \tilde{M}=-\frac{3}{k \nu} \frac{\left(m^{4}-m^{2}+1\right)^{5 / 2}}{\left(s_{1}^{2}-3 s_{2}\right)^{3 / 2} m^{3}\left(m^{2}-1\right)} \tilde{M}
$$

where

$$
\tilde{M}=2\left(\frac{1}{2 P} \int_{-P}^{P} u_{0} \bar{V}\left(u_{0}\right) d \theta\right)
$$

The differential equation (30) describes the slow modulations of the cnoidal wave (12) as it propagates with constant speed $c$, for some given $\bar{V}$ (where $\theta=\Theta / \epsilon$ ). Now, to concentrate on the traffic problem detailed in Section $2, \bar{V}$ is defined using (8). So then

$$
\begin{aligned}
\tilde{M}= & -\left(\mu k^{2}+2 U \eta k^{2}\right) \frac{4 \nu}{5 \lambda k^{2}}(3 \hat{D}+2 \hat{C} d+U(\hat{C}+U d)) \\
& +\left(\gamma k^{4}-\frac{2 \lambda \eta}{\nu} k^{4}\right) \frac{2 \nu^{2}}{7 \lambda^{2} k^{4}}\left(6 \hat{D} d+8 \hat{C}^{2}+U\left(-6 \hat{D}+6 \hat{C} d+2 U \hat{C}+2 U^{2} d\right)\right) .
\end{aligned}
$$

As we seek solutions that satisfy periodic boundaries, one way to achieve this is to set $m_{\Theta}=0$, and therefore, $\tilde{M}=0$. As a result, the modulation term $m$, and therefore, the wave amplitude, 
period and mean height will remain constant over the solution domain. The choice of constant $m$ is motivated by the numerical simulations conducted by Zhu and Dai (2008), Yu et al. (2010) and Zhou et al. (2014) already discussed. Hattam and Clarke (2015) also used this technique of fixing $m$ to obtain spatially periodic solutions to the periodically forced steady KdV-Burgers equation. The period of these solutions was equal to or an integer multiple of the forcing term's period.

Now, let us express our parameters in terms of $m, s_{1}$ and $\kappa$ such that

$$
a=\sqrt{\kappa} H_{1}(m), \quad d=\frac{s_{1}}{6}+\sqrt{\kappa} H_{3}(m), \quad \hat{C}=\kappa-\frac{s_{1}^{2}}{72}, \quad \hat{D}=\kappa^{3 / 2} H_{2}(m)-\frac{s_{1}}{6}\left(\kappa-\frac{s_{1}^{2}}{216}\right),
$$

where

$$
\begin{aligned}
& H_{1}(m)=\left(\frac{18 m^{4}}{1-m^{2}+m^{4}}\right)^{1 / 2} \\
& H_{2}(m)=\frac{H_{1}(m)^{3}}{162 m^{6}}\left(-2+3 m^{2}+3 m^{4}-2 m^{6}\right), \\
& H_{3}(m)=\frac{H_{1}(m)}{3 m^{2}}\left(\frac{3 E(m)}{K(m)}+m^{2}-2\right)
\end{aligned}
$$

Substituting the definitions given by (32) into (31) and simplifying, (31) reduces to

$$
\tilde{M}=-2\left(\mu+\frac{s_{1}}{3} \eta\right) \frac{2 \nu \kappa^{3 / 2}}{5 \lambda}\left(3 H_{2}(m)+2 H_{3}(m)\right)+2\left(\gamma-\frac{2 \lambda \eta}{\nu}\right) \frac{\nu^{2} \kappa^{2}}{7 \lambda^{2}}\left(6 H_{2}(m) H_{3}(m)+8\right) .
$$

Therefore, to ensure $m_{\Theta}=0$, then

$$
-\left(\mu+\frac{s_{1}}{3} \eta\right) \frac{2 \nu \kappa^{3 / 2}}{5 \lambda}\left(3 H_{2}(m)+2 H_{3}(m)\right)+\left(\gamma-\frac{2 \lambda \eta}{\nu}\right) \frac{\nu^{2} \kappa^{2}}{7 \lambda^{2}}\left(6 H_{2}(m) H_{3}(m)+8\right)=0 .
$$

If (33) holds then the leading order solution will be periodic since it becomes the cnoidal wave of constant modulus $m$ i.e. the period, amplitude and mean value remain unchanged for all $\theta$.

Given $m$ and $c$ are some constants, then $\omega$ and $k$ will also be constants $(\Theta=k X-\omega T)$, where

$$
k(m)=\sqrt{\frac{\nu a(m)}{12 \lambda}} \frac{P}{m K(m)} .
$$

This gives, with (25),

$$
\kappa=\frac{s_{1}^{2}}{18}-\frac{s_{2}}{6}=\left(\frac{k K(m)}{P}\right)^{4}\left(\frac{12 \lambda}{\nu}\right)^{2} \frac{\left(m^{4}-m^{2}+1\right)}{18} .
$$

Rearranging (33) and using (35), we obtain

$$
s_{1}=\frac{180}{7 \bar{\rho}(m)}\left(\frac{k}{P}\right)^{2}\left(\frac{\gamma}{\eta}-\frac{2 \lambda}{\nu}\right)-\frac{3 \mu}{\eta},
$$


where

$$
\bar{\rho}(m)=\frac{H_{1}(m)}{(m K(m))^{2}}\left(\frac{3 H_{2}(m)+2 H_{3}(m)}{3 H_{2}(m) H_{3}(m)+4}\right) .
$$

This relation determines the speed $c=\left(\nu s_{1}\right) / 6$ of our cnoidal wave solutions for some choice of fixed $m,(k / P)$ and equation parameters $\lambda, \nu, \mu, \gamma, \eta$. Hence, a family of periodic solutions to (7)-(8) exist in the limit $0<\epsilon \ll 1$ when (36) holds.

\section{Application to the Traffic Flow Model}

The analysis outlined in Sections 3 and 4 is now related to the traffic flow model (2). It is therefore necessary to express the leading order solution in terms of the model (2) variables, $j$ and $t$. As well, the equation parameters $\lambda, \nu, \mu, \gamma, \eta$ are now defined using (9).

Without any loss of generality, we firstly set $k=1(\theta=x)$ and then, $c=\omega$. So, our travelling wave solution in terms of car $j$ 's headway at time $t$ is

$$
\Delta x_{j}(t)=h+\frac{\epsilon^{2}}{V^{\prime \prime}(h)} u_{0}(j, t)+O\left(\epsilon^{3}\right),
$$

where, using (5),

$$
\begin{aligned}
u_{0}(j, t)=a( & m) b(m)+d(m) \\
& +a(m) \operatorname{cn}^{2}\left(\frac{K(m)}{P}\left(\sqrt{12\left(\tau_{s}-\tau\right)}\left(j+t\left(V^{\prime}(h)+\frac{s_{1}}{6}\left(\frac{\tau_{s}-\tau}{\tau_{s}}\right)\right)\right)+\theta_{0}\right) ; m\right) .
\end{aligned}
$$

The solution parameter $s_{1}$ is chosen such that (36) is satisfied and $m$ is fixed. The solution parameters $b, a$ and $d$ are defined by (13c), (25) and (27) respectively. The speed of this wave is

$$
\text { wave speed }=V^{\prime}(h)+\frac{s_{1}}{6}\left(\frac{\tau_{s}-\tau}{\tau_{s}}\right) .
$$

Note that the solutions highlighted in Section 4 are periodic in the $\theta$ direction, however, they are not necessarily periodic over the spatial domain $j \in[0, N]$. Let us set

$$
n \hat{T}=N
$$

If $n$ is equal to some positive integer then the corresponding solution satisfies periodic boundaries. Consequently, $n$ is the number of oscillations over $j \in[0, N]$ and the solution period in the $j$ direction is $\hat{T}=(2 P) / \sqrt{12\left(\tau_{s}-\tau\right)}$. Hence,

$$
P=\frac{N}{2 n} \sqrt{12\left(\tau_{s}-\tau\right)} .
$$


The periodic boundary conditions (10) hold if $P$ is defined using (40), where $n=1,2, \ldots$

There exist a large number of possible travelling wave solutions that satisfy (36) and (40). To restrict our analysis, we will seek solutions similar to that found numerically by Zhu and Dai (2008), such that

$$
\Delta x_{0, N}\left(t_{0}\right)=h, \quad \frac{\partial \Delta x_{0, N}\left(t_{0}\right)}{\partial j}=0,
$$

and therefore,

$$
u_{0}\left(j=0, N ; t=t_{0}\right)=0, \quad \frac{\partial u_{0}}{\partial j}\left(j=0, N ; t=t_{0}\right)=0,
$$

where we set $t_{0}=0$ without any loss of generality.

So that (41) is met, let us set $\theta_{0}=P\left(\theta_{0}\right.$ is arbitrary at leading order $)$ and $a b+d=0$, which gives, using (25) and (27),

$$
s_{1}(m)=-6\left(a(m) b(m)+\frac{a(m)}{3 m^{2}}\left(3 \frac{E(m)}{K(m)}+m^{2}-2\right)\right) .
$$

Now, combining (36) and (42), and after some manipulation, we arrive at a definition for the driver's delay time,

$$
\tau=\frac{2 n^{2}}{3 N^{2} V^{\prime}(h)}\left(\frac{15}{7 \bar{\rho}(m)}+6 m^{2} K(m)^{2}\left(b+\frac{1}{3 m^{2}}\left(\frac{3 E(m)}{K(m)}+m^{2}-2\right)\right)\right)+\frac{1}{2 V^{\prime}(h)} .
$$

So, $\tau$ and therefore $\hat{a}=1 / \tau$ (refer to (2)) are dependent upon $(n / N), m$ and $V^{\prime}(h)$. Figure 1 depicts the driver's sensitivity $\hat{a}$ as a function of $m$ for $h=3.5$ or 4.5 (left) and $h=2.5$ or 5.5 (right). Each curve signifies when a cnoidal wave solution satisfying (41) occurs for some value of integer $n$ and when $N=100$ (for 100 cars on the road). Only when $\hat{a}>\hat{a}_{s}$ will a solution exist $(\epsilon>0)$. It is apparent that as $\hat{a}$ grows, the modulation term $m$ increases for some fixed $n$.

From (42) and (43), it is evident that the wave speed (39) is determined by also specifying $(n / N)$, $m$ and $V^{\prime}(h)$. The plot of the wave speed is displayed in Figure 2 for $h=3.5$ or 4.5 (left) and $h=2.5$ or 5.5 (right) as a function of the modulus $m$, with each curve corresponding to some choice of $n$ and $N=100$. This figure reveals that the wave speed increases with $m$ for some choice of $n$. Hence, as the driver's sensitivity becomes larger, the traffic density wave propagates with greater speed.

\section{$6 \quad$ Stability}

The spatially periodic asymptotic solutions, given by (37)-(38), are plotted in Figures 3-7. So that (41) holds, the parameters $P, s_{1}$ and $\tau$ are defined using (40), (42) and (43) respectively. Now that the relation between the solution properties and the driver's sensitivity $\hat{a}$ is established, the OV 

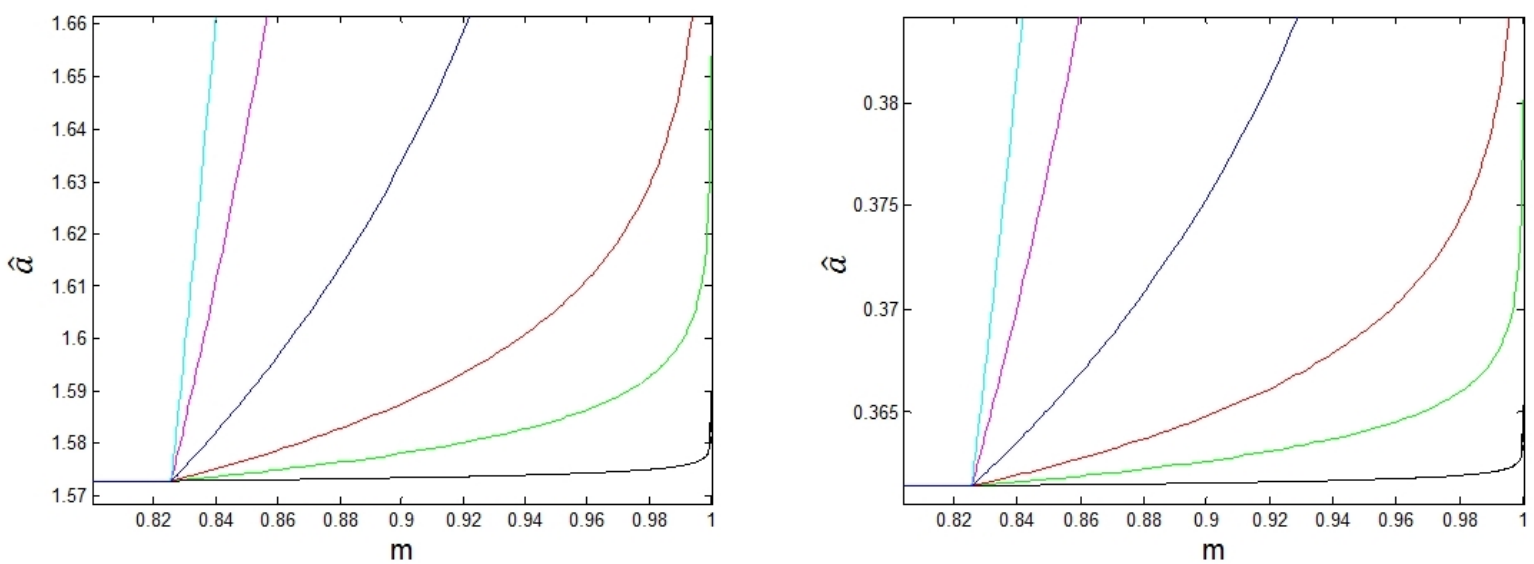

Figure 1: The drivers sensitivity, $\hat{a}$, which appears in the OV model (2), is determined by $m$ and $n$. Each curve represents $\hat{a}(m)=1 / \tau(m)$ for some choice of $n$, where $\tau$ is given by (43), $N=100$, black: $n=1$, green: $n=3$, red: $n=5$, blue: $n=10$, purple: $n=20$, light blue: $n=30$. Left: $h=3.5,4.5, \hat{a}_{s}=1.5729$. Right: $h=2.5,5.5, \hat{a}_{s}=0.36141$.
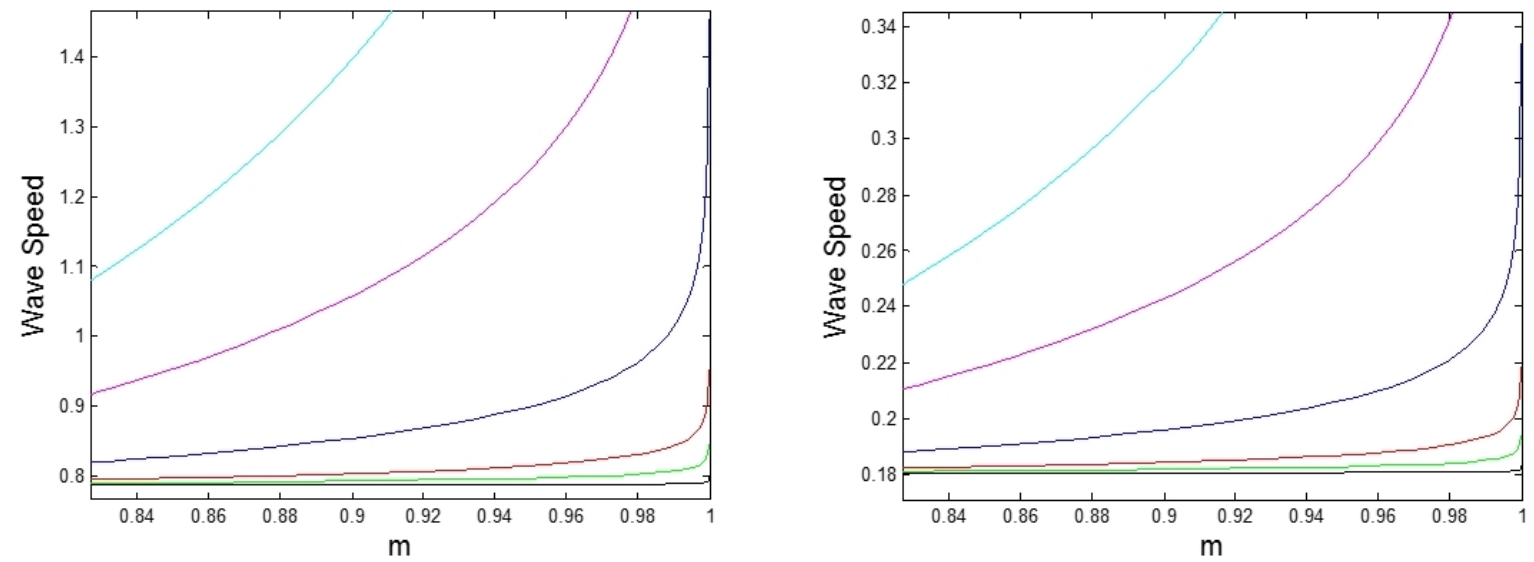

Figure 2: The wave speed (39) of the cnoidal wave solution (38) as a function of the modulus m, for some choice of $n$, where $N=100$, black: $n=1$, green: $n=3$, red: $n=5$, blue: $n=10$, purple: $n=20$, light blue: $n=30$. Left: $h=3.5,4.5$. Right: $h=2.5,5.5$. 
model (2) can be solved with MATLAB's ode45, and then the asymptotic and numerical results compared. Periodic boundary conditions (10) are also imposed. The initial condition used for the simulation is defined by (37)-(38) at $t=0$. As well, the comparisons between the asymptotic and the numerical solution are performed over varying time intervals, with their long-time behaviour examined.

In Figure 3, the solution for $h=3.5, \hat{a}=1.59, \epsilon=0.10372, N=100$ and $n=1$ is shown, where an upward density wave is depicted. The top panel of Figure 3 compares the asymptotic solution, given by (37)-(38), to the numerical findings for $t \in[0,100]$. The middle panel displays the headway profile for car $j=0, N$, where the solid black curve represents the asymptotic solution and the dotted red curve is the ode45 solution. These plots suggest excellent agreement. Next, the simulation is solved over a large time domain to examine numerical stability. The result is given along the bottom panel of Figure 3 (at around $t=1000$ and $t=10000$ ), where the headway profiles for car $j=0, N$ is displayed. It is evident that numerically, the density wave persists for a considerably long time, although eventually a slight phase shift and a reduction in amplitude develops. It will eventually disappear. This is expected since all the depicted solutions satisfy the linear stability criteria (4). Hence, all disturbances will dissolve as $t \rightarrow \infty$ and $\Delta x_{j}$ will tend to steady state $h$, as discussed in Section 2. These observations are consistent with the numerical findings of Muramatsu and Nagatani (1999) for solutions in the metastable zone, although they considered open boundaries.

Next, in Figure 4, the driver's sensitivity is increased to $\hat{a}=1.65(\epsilon=0.21617)$, and consequently, the solution is larger in amplitude, with a narrower peak (since $m$ is increased to be extremely close to 1). This is of a form similar to a soliton, although, as it is actually a cnoidal wave, the periodic boundary conditions are satisfied. Zhu and Dai (2008) found numerically solutions of this form when they considered periodic boundaries, however they referred to these as solitons. The top panel compares the numerical and asymptotic findings for $t \in[0,100]$. The bottom panel depicts the headway profiles of car $j=0, N$ for $t \in[0,100]$ (left) and $t \in[800,1000]$ (right). The asymptotic and numerical solutions are in good agreement. However, as $\epsilon$ is larger, the excellent match observed for $\hat{a}=1.59$ is not achieved here. Moreover, as a result of increasing $\epsilon$, the solution will disappear significantly faster since a notable phase shift and amplitude reduction appears around $t=900$. This behaviour can be attributed to a greater wave speed.

In Figure 5, again solutions for $n=1, N=100$ are examined except now $h=4.5$. The same choice of $m$ and wave speed used for $h=3.5$ will apply here. The top panel relates to $\hat{a}=1.59$ $(\epsilon=0.10372)$ and the bottom to $\hat{a}=1.65(\epsilon=0.21617)$. Asymptotic solutions for $t \in[0,100]$ are shown on the left, and on the right, the asymptotic solutions for car $j=0, N$ are compared to the numerical findings. The agreement between these two solutions and the long-time behaviour are 


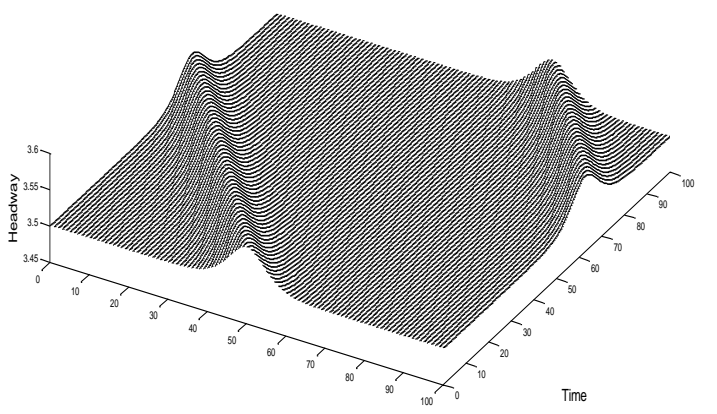

Car Number

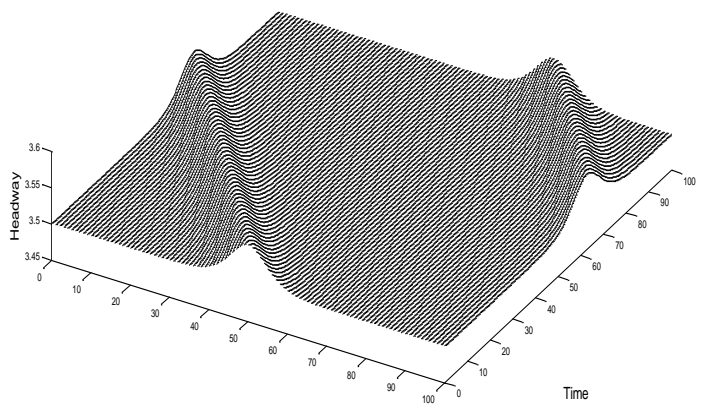

Car Number
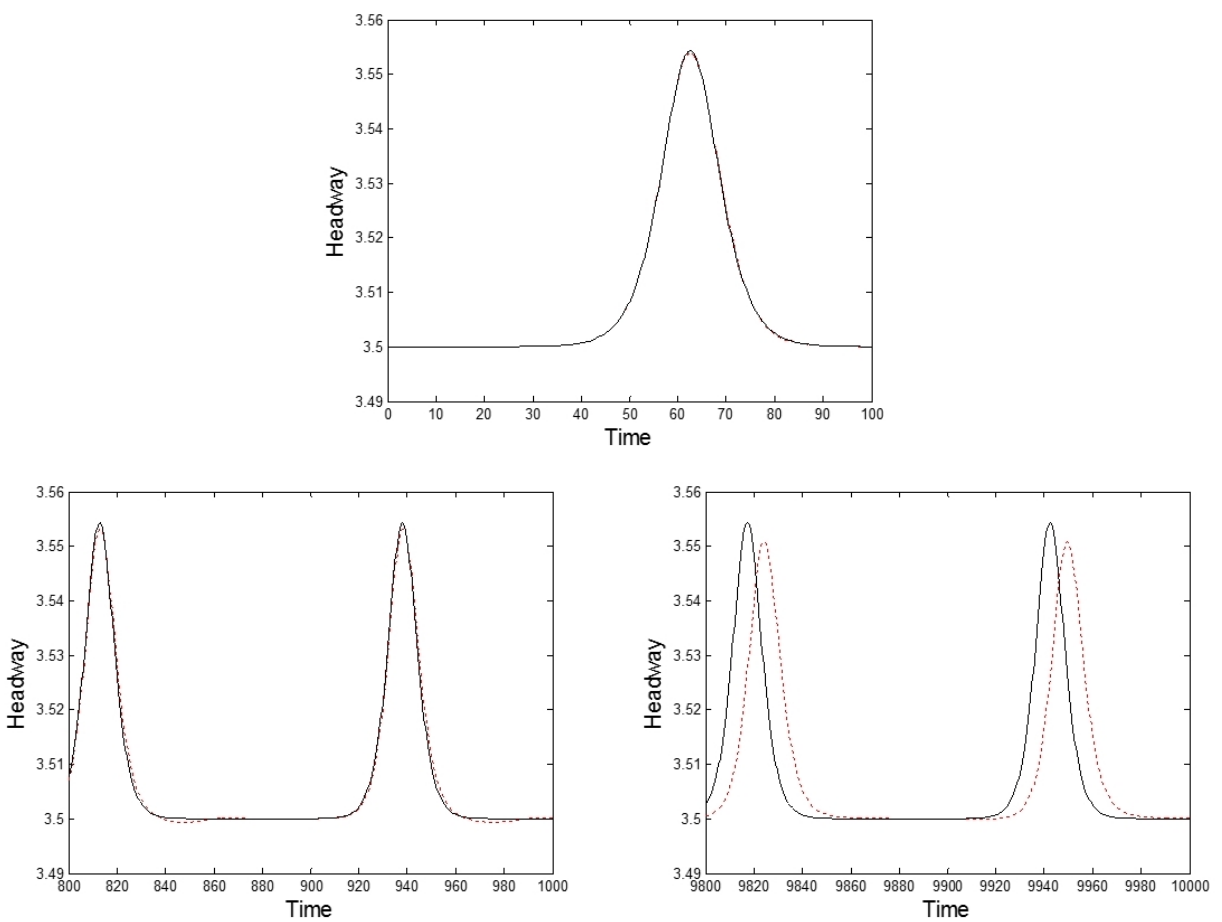

Figure 3: Spatially periodic headway solutions for cars $j=0,1, \ldots, 100$, with $h=3.5, \hat{a}=1.59, \epsilon=0.10372$, $m=0.999998947, n=1$, wave speed=0.79961. Top left: Asymptotic headway solution given by (37)-(38). Top right: MATLAB ode45 headway solution to (2) with the initial condition defined by (37)-(38). Middle: Headway profile for car $j=0,100$, where the asymptotic solution corresponds to the solid black curve and the ode 45 solution is represented by the dotted red curve. Bottom: Headway profile for car $j=0,100$ and $t \in[800,1000]$ (left), $t \in[9800,10000]$ (right), where the asymptotic solution corresponds to the solid black curve and the ode 45 solution is represented by the dotted red curve. 

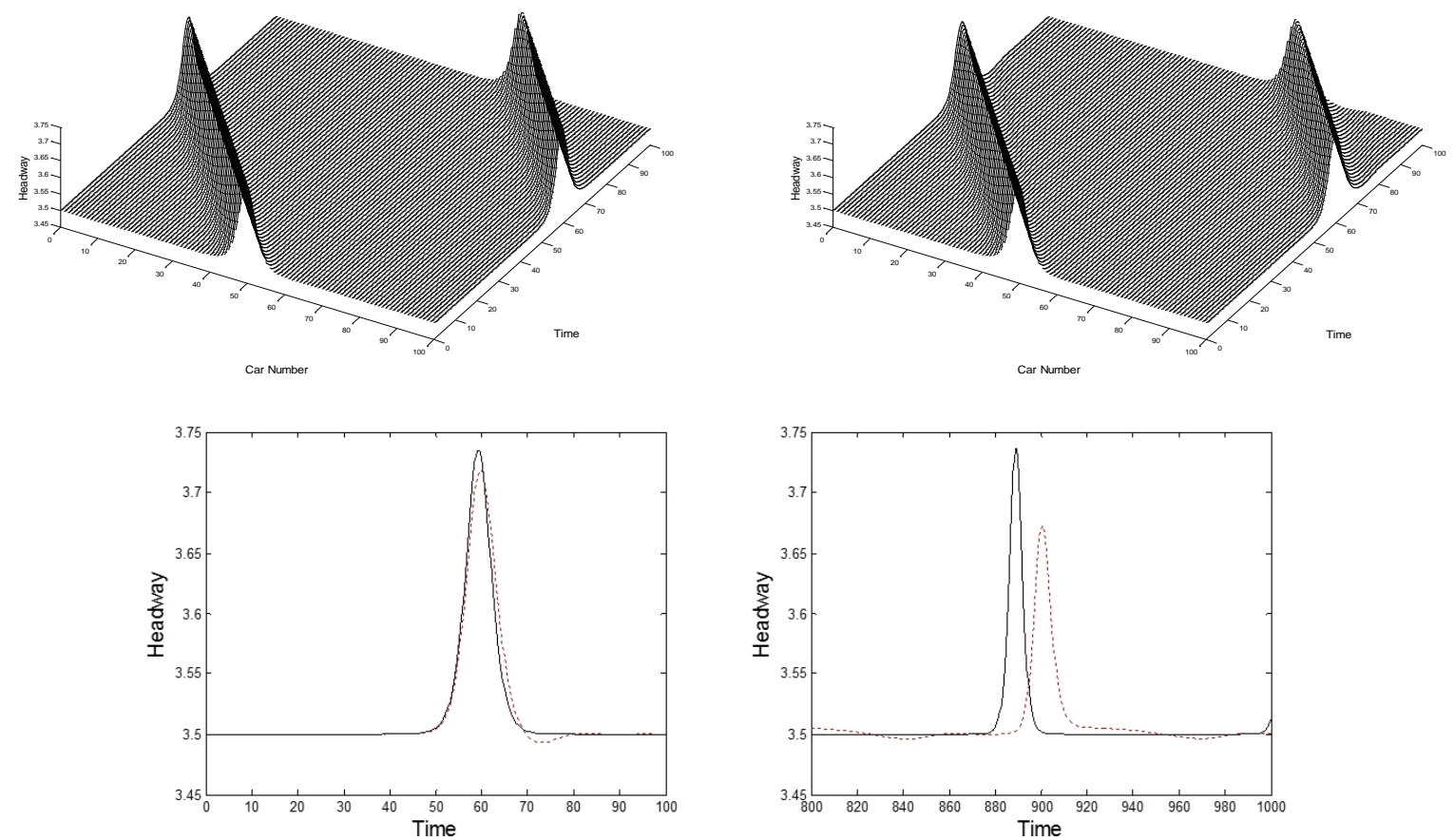

Figure 4: Spatially periodic headway solutions for cars $j=0,1, \ldots, 100$, with $h=3.5, \hat{a}=1.65, \epsilon=0.21617$, $m=0.999999999999963, n=1$, wave speed $=0.84357$. Top left: Asymptotic headway solution given by (37)-(38). Top right: MATLAB ode45 headway solution to (2) with the initial condition defined by (37)(38). Bottom: Headway profile for car $j=0,100$ and $t \in[0,100]$ (left), $t \in[800,1000]$ (right), where the asymptotic solution corresponds to the solid black curve and the ode 45 solution is represented by the dotted red curve. 

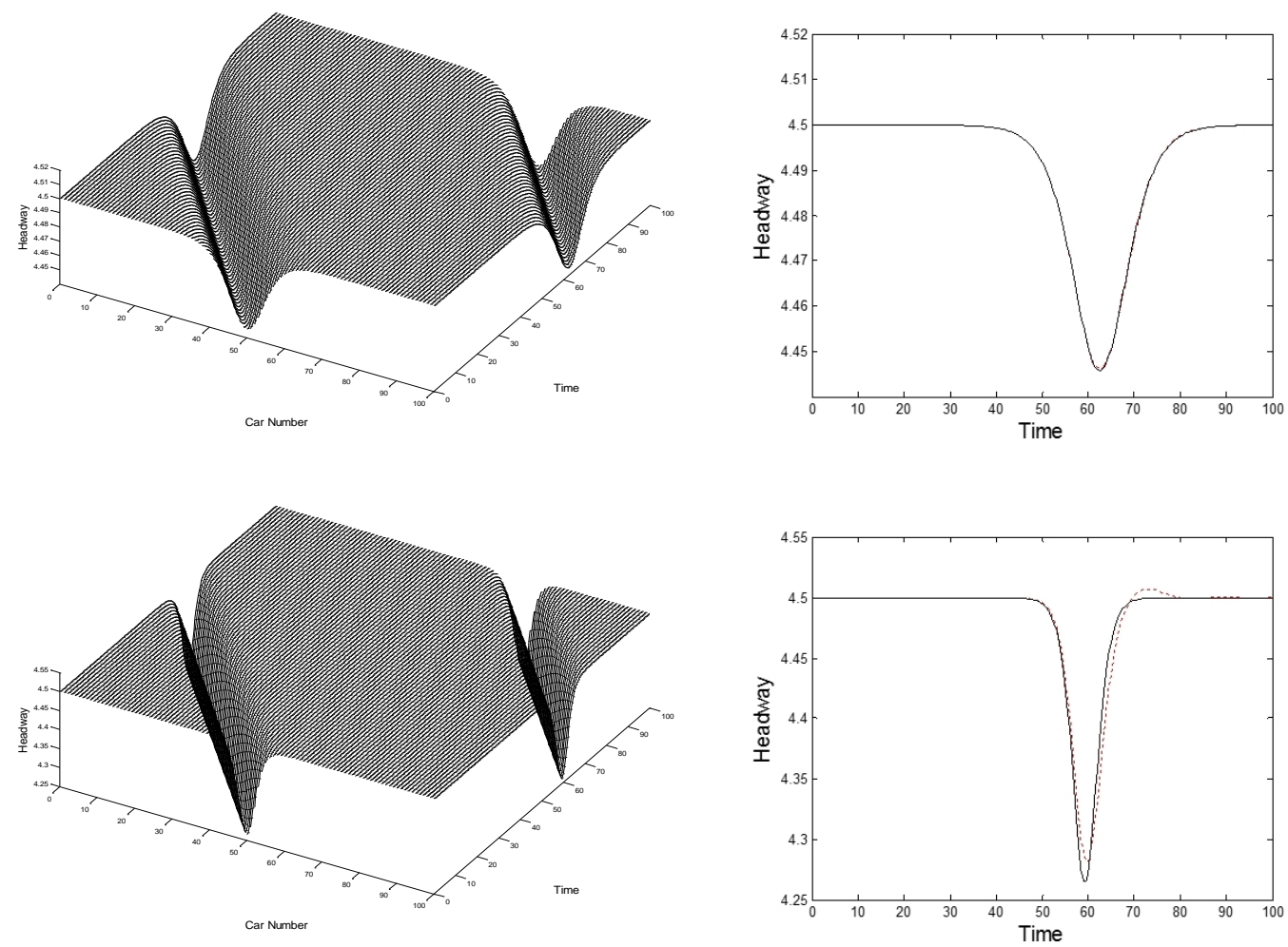

Figure 5: Spatially periodic headway solutions for cars $j=0,1, \ldots, 100$, with $h=4.5, n=1$. Top: $\hat{a}=1.59, \epsilon=0.10372, m=0.999998947$, wave speed $=0.79961$. Bottom: $\hat{a}=1.65, \epsilon=0.21617, m=$ 0.999999999999963, wave speed $=0.84357$. Top/Bottom left: Asymptotic headway solution given by (37)(38). Top/Bottom right: Headway profile for car $j=0,100$, where the asymptotic solution corresponds to the solid black curve and the ode 45 solution to (2) is represented by the dotted red curve.

consistent with the discussions for $h=3.5$. However, now the solution is a downward form density wave since $h>h_{c}=4$. Zhu and Dai (2008) made similar observations, explaining that if $h<h_{c}$, vehicle $j$ slows down to avoid crashing into vehicle $j+1$. As a result, an upward form density wave occurs. Whereas, if $h>h_{c}$, vehicle $j$ speeds up to the maximal velocity and consequently, a downward form density wave emerges. Given the traffic density is defined as the inverse of the headway, then the upward and downward waves represent clusters of faster and slower moving vehicles respectively. Hence, downward waves represent traffic congestion.

Now, we investigate the solutions with $n=2, h=3.5, N=100$ so that two density waves propagate, see Figure 6. The top panel corresponds to $\hat{a}=1.59(\epsilon=0.10372)$, and the bottom to $\hat{a}=1.65(\epsilon=0.21617)$. As exhibited when $n=1$, upward and downward waves form when $h<h_{c}$ and $h>h_{c}$ respectively, although solutions with $h>h_{c}$ are not depicted here. On the left, the 


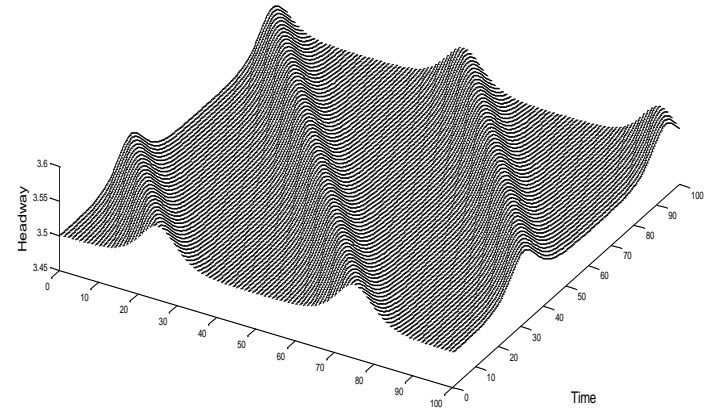

Car Number

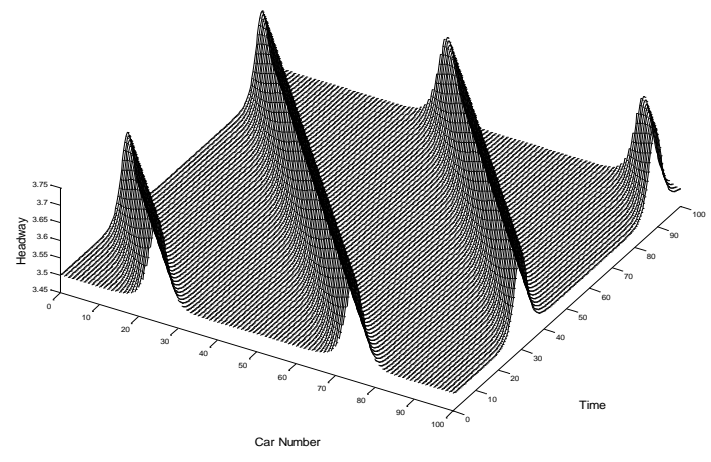

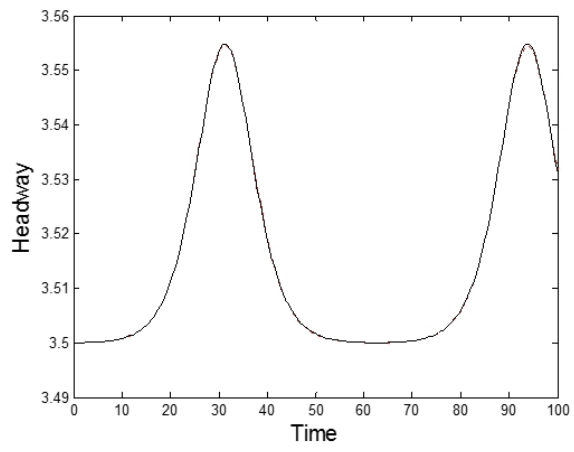

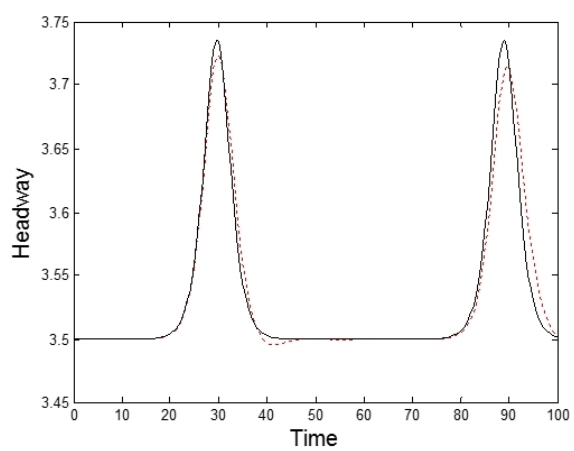

Figure 6: Spatially periodic headway solutions for cars $j=0,1, \ldots, 100$, with $h=3.5, n=2$. Top: $\hat{a}=1.59$, $\epsilon=0.10372, m=0.99724797$, wave speed=0.79967. Bottom: $\hat{a}=1.65, \epsilon=0.21617, m=0.99999946$, wave speed $=0.84362$. Top/Bottom left: Asymptotic headway solution given by (37)-(38). Top/Bottom right: Headway profile for car $j=0,100$, where the asymptotic solution corresponds to the solid black curve and the ode45 solution to (2) is represented by the dotted red curve.

asymptotic solution over the domain $t \in[0,100]$ is displayed and on the right, a comparison between the numerical and asymptotic findings for car $j=0, N$ is given. There is good agreement between the two results, especially when $\epsilon=0.10372$, which is expected. Once again, these travelling wave solutions will eventually dissolve at very large $t$, with the $\hat{a}=1.59$ result persisting for a far greater time due to a smaller wave speed. Furthermore, the amplitude is notably larger and narrower for $\epsilon=0.21617$, which is a result of increasing $m$.

Such studies as Yu et al. (2010) highlighted numerical density waves with multiple oscillations over the same spatial domain. To obtain this solution type, $n$ can continue to be increased. Analysing these solutions, it becomes apparent that as $n$ grows, for some fixed $\hat{a}$, the amplitude and wave speed slightly increases. As well, these solutions are found to have a similar long-time behaviour as that demonstrated for $n=1$. As an example, Figure 7 depicts an asymptotic solution with three 

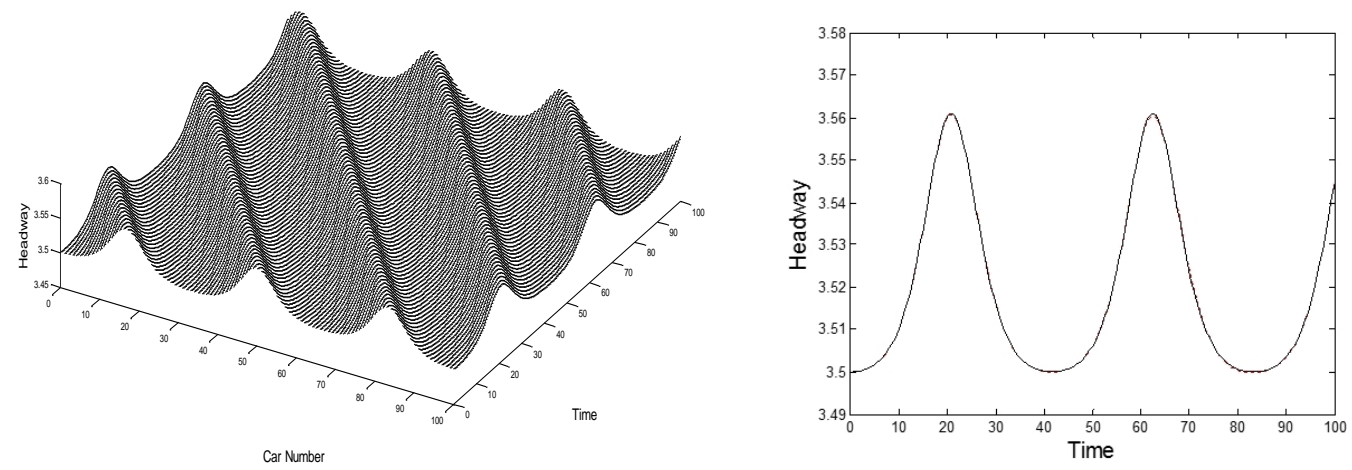

Figure 7: Spatially periodic headway solutions for cars $j=0,1, \ldots, 100$, with $h=3.5, \hat{a}=1.59, \epsilon=0.10372$, $n=3, m=0.9728972$, wave speed $=0.80039$. Left: Asymptotic headway solution given by (37)-(38). Right: Headway profile for car $j=0,100$, where the asymptotic solution corresponds to the solid black curve and the ode 45 solution to (2) is represented by the dotted red curve.

oscillations and where $\hat{a}=1.59, N=100, h=3.5$.

\section{Conclusion}

The evolution of traffic behaviour was determined using the OV model (2). Previous studies have examined this model numerically and analytically, with the stability regimes well-outlined (refer to Ge et al. (2005)). Here, we concentrated on the metastable zone that corresponded to the onset of traffic jams and where (2) reduced to the perturbed KdV equation (7). Modulation theory detailed by Myint and Grimshaw (1995) and Kamchatnov (2004) was then applied to (7). As a result, at leading order, the cnoidal wave solution was obtained and at the next order, the Whitham system was derived, which was altered due to the perturbation terms of (7). Therefore, all possible solutions due to modulation theory were identified. Next, we chose to focus on a particular solution type, where steady travelling wave solutions were sought by ensuring the wave speed remained constant. Consequently, the three Whitham equations were transformed into a single equation for the slow variation of the modulation term, $m$, defined by (30). Setting (30) to zero so that $m$ was fixed over the solution domain then led to the identification of periodic cnoidal wave solutions. The resulting solutions had constant amplitude, mean height and period, which were of a similar form to the numerical waves depicted by, for instance, Yu et al. (2010). This analysis was next applied to the traffic flow problem by defining the leading order solution in terms of car $j=1, \ldots, N$ and imposing the periodic boundary conditions along $j \in[0, N]$. Thus, a family of travelling 
wave solutions were highlighted, where the choice of $m$ and the number of oscillations over the spatial domain were shown to determine the driver's sensitivity, $\hat{a}$, and the wave speed. Due to determining these relationships, numerical solutions of the OV model were calculated and compared to the asymptotic headway solutions. Overall, a good agreement between the two solutions was observed. Moreover, the long-time dynamics were explored. This revealed that the numerical density waves dissolved after a considerable length of time, which was consistent with the linear stability analysis detailed in Section 2. This paper has provided an extension of other workings that only considered traffic soliton solutions (for example see Muramatsu and Nagatani (1999) and Zhu and Dai (2008)). Instead here modulation theory was used to establish the existence of cnoidal waves in the traffic model (2), with the wave properties related to the model parameters.

\section{References}

M. Muramatsu, T. Nagatani, Soliton and kink jams in traffic flow with open boundaries, Phys. Rev. E 60 (1999) 180-187.

T. Nagatani, The physics of traffic jams, Rep. Prog. Phys. 65 (2002) 1331.

G. Newell, Nonlinear effects in the dynamics of car following, Oper. Res. 9 (1961) 209-229.

D. Korteweg, H. de Vries, On the change of form of long waves advancing in a rectangular canal, and on a new type of long stationary waves, Phil. Mag. 39 (1895) 422-443.

H. Zhu, S. Dai, Numerical simulation of soliton and kink density waves in traffic flow with periodic boundaries, Physica A 387 (2008) 4367-4375.

L. Yu, T. Li, Z.-K. Shi, Density waves in a traffic flow model with reaction-time delay, Physica A 389 (2010) 2607-2616.

J. Zhou, Z.-K. Shi, J.-L. Cao, An extended traffic flow model on a gradient highway with the consideration of the relative velocity, Nonlinear Dynam. 78 (2014) 1765-1779.

G. Whitham, Linear and Nonlinear Waves, Wiley, New York, 1974.

A. Gurevich, L. Pitaevskii, Averaged description of waves in the Korteweg-de Vries-Burgers equation, Sov. Phys.-JETP 66 (1987) 490-495.

S. Myint, R. Grimshaw, The modulation of nonlinear periodic wave-trains by dissipative terms in the Korteweg-de Vries equation, Wave Motion 22 (1995) 215-238. 
A. Kamchatnov, On Whitham theory for perturbed integrable equations, Physica D 188 (2004) $247-261$.

M. Bando, K. Hasebe, A. Nakayama, A. Shibata, Y. Sugiyama, Dynamical model of traffic congestion and numerical simulation, Phys. Rev. E 51 (1995) 1035-1042.

H. Ge, R. Cheng, S. Dai, KdV and kink-antikink solitons in car-following models, Physica A 357 (2005) 466-476.

G. El, R. Grimshaw, A. Kamchatnov, Analytic model for a weakly dissipative shallow-water undular bore, Chaos 15 (2005) 13.

L. Hattam, S. Clarke, Modulation theory for the steady forced KdV-Burgers equation and the construction of periodic solutions, Wave Motion 56 (2015) 67-84. 